\title{
Minimizing Nurses Psychological Conflict over Physical Restraint of Patients
}

Miwako Tokunaga, Yoshikazu Tominaga, Natsuko Koga and Takeharu Koga*

Asakura Medical Association Hospital, Asakura, Japan

*Corresponding author: Takeharu Koga, Asakura Medical Association Hospital, Asakura, Japan; Tel: +81-946-23-0077; Fax: +81-946-23-0076; E-mail: koga.tk@asakura-med.or.jp

Received date: Jan 19, 2015; Accepted date: Mar 31, 2015; Published date: Apr 7, 2015

Copyright: ( $) 2015$ Tokunaga $M$ et al. This is an open-access article distributed under the terms of the Creative Commons Attribution License, which permits unrestricted use, distribution, and reproduction in any medium, provided the original author and source are credited.

\section{Abstract}

Physical restraint (PR) is a widespread practice in many countries, and that nurses play a key role in the decisionmaking and practicing PR with a substantial psychological conflicts. Although little is understood how the conflicts should be controlled and integrated into a safe and ethical management of patients, several aspects of PR are becoming clearer which may aid dealing with its use.

Keywords: Physical restraint; Conflict; Nurse

\section{Commentary}

Physical restraint (PR) is defined as a mechanical or protective device applied to limit the free movement of patients [1]. Although PR may be inevitable to secure devices critical to the patients' life such as endotracheal tubes, or certain drainage or infusion lines, its use raises not only ethical issues but also entails direct known detrimental effects to the patients such as increased risks for developing post-traumatic stress disorder [2] and delirium [3]. Unfortunately, PR is not always effective for its intended use, as illustrated by the fact that PR is associated with an increased risk of unplanned extubation [4]. Therefore, it is widely appreciated that use of PR should be limited and minimized.

Many nurses around the world must have ambivalent feelings about $\mathrm{PR}$. On the one hand PR is an essential procedure to secure the treatment and safety of patients, and on the other hand it is associated with difficult ethical issues. This ambivalent feeling, defined as psychological conflict, appears to be prevalent among practicing nurses.

Psychological conflict can be a significant burden on nurses, who judge must judge when to use PR, and this burden may cause some nurses even to leave the profession [5]. A nation-wide survey in Japan reported that PR was applied in $25.5 \%$ of patients in chronic hospitals, and that it exceeded 30 days in most of the restrained patients [6]. In European countries, patients in ICUs were not restrained at all in England and Portugal, while about half of the patients were restrained in Swiss, Spain, and France, and $100 \%$ in in Italy [7]. A recent survey of ICUs in France found that $82 \%$ of the patients were restrained, and that PR was applied even in $30 \%$ of patients who were conscious and appeared able to obey orders [8]. The survey also disclosed that the restriction was initiated or terminated without written orders in most of the cases [8]. These results suggest that the prevalence of PR varies by institution or by region, and is generally high in ICUs. Also, its application often depends primarily on nurses' judgment.

PR provokes psychological conflict in $36.4 \%$ of attending nurses in ICUs, a survey reported in 2014 [9]. A majority of nurses is likely to have ethical dilemmas concerning the harm and benefits of PR during application. On the other hand, in most cases PR is intended primarily to ensure the security of patients, including other patients [10]. Is there any way to reduce this conflict, aside from eliminating PR

The aforementioned point prevalence survey across ICUs in Europe [7], which found that thirty-three percent of all patients were physically restrained, also documented that the prevalence of PR varied from 0 to $100 \%$. Focusing on the nurse-to-patient ratio, it was 1:1 England and Portugal, where PR was not used. On the other hand, the ratio in France, where PR is more prevalent, was 1:2 in five hospitals, 1:3 in six, and 1:4 in one institution, which were all lower than that of England and Portugal. The ratio in Italy, where all patients were restrained, was 1:2, suggesting that the nurse-to-patient ratio alone cannot explain the difference in the prevalence of PR. The position statement from the British Association of Critical Care Nurses [11] is intended to facilitate optimal patient care. It stresses that PR should not be an alternative to insufficient human or environmental resources. According to the statement, application of $\mathrm{PR}$ is to be determined by team consensus with the participation of the patient and their family, and execution is to be based on specific protocols and/or guidelines. Education of all staff regarding PR constitutes an important part of their program. These elements appear to be useful strategies to reduce the conflict of practicing nurses.

Intubated patients are more likely than others to undergo PR, since self- or accidental extubation can be directly life threatening. A casecontrol study of 100 patients with unplanned extubation disclosed that an impaired level of consciousness on admission to the intensive care unit and the presence of nosocomial infection intensify the risk for unplanned extubation, even when physical restraints are used [4]. Other risk factors associated with unplanned extubation include male gender, presence of restlessness/agitation, lower sedation level/higher consciousness levels, and severity of physiological impairment, and PR [12]. Analysis of the 151 patients in the ICU with unplanned extubation in our institution identified dementia or cognitive impairment as a risk, and sedatives and/or hypnotics before sleep were protective factor for unplanned extubation (unpublished observation). On the other hand, weaning protocols from mechanical ventilation were associated with decreased incidence of unplanned extubation [13]. These observations suggest that certain clinical situations are associated with an increased risk of unplanned extubation, and that some measures could be implemented to minimize PR. For instance, security of sleep for inpatients, particularly for those with cognitive 
dysfunction, prevention of delirium, and promotion of weaning from a ventilator if ventilated could help to minimize the use of PR. Establishing a decision-making protocol for the initiation, continuation, and cessation of PR can be a critical element to reduce nurses' conflict over PR. A review of this process pointed out those context- and nurse-related factors can hinder nurses from making an ethical decision on the appropriate use of physical restraints [9]. The former denotes the balancing of ethical values and safety issues, and the latter refers to nurses' interpretation of patient behavior and personal relationships between nurses and their patients, which may create the opportunity for nurses to approach each person as a unique individual with respect to his or her dignity and autonomy. To deal with these aspects, the review stresses the importance of a valuesupportive environment, which is essential for developing the transformational leadership style and ethical leadership capacities of each nurse. In addition, organizing a process and/or a team which aids and/or advises practicing nurses in the decision-making process would be a means of reducing the psychological conflicts of nurses.

In conclusion, $\mathrm{PR}$ is a widespread practice in many countries.Nurses play a key role in the decision-making and execution of PR, and this can cause a substantial psychological conflicts.However, little is understood about how these conflicts should be controlled and integrated for the safe and ethical management of patients, although several aspects of PR are becoming clearer which may aid in minimizing its use. Focused researches on this topic are warranted.

\section{References}

1. Scherer YK, Janelli LM, Kanski GW, Neary MA, Morth NE (1991) The nursing dilemma of restraints. J Gerontol Nurs 17: 14-17.

2. Jones C, Bäckman C, Capuzzo M, Flaatten H, Rylander C, et al. (2007) Precipitants of post-traumatic stress disorder following intensive care: a hypothesis generating study of diversity in care. Intensive Care Med 33: 978-985.

3. Van Rompaey B, Elseviers MM, Schuurmans MJ, Shortridge-Baggett LM, Truijen S, et al. (2009) Risk factors for delirium in intensive care patients: a prospective cohort study. Crit Care 13: R77.

4. Chang LY, Wang KW, Chao YF (2008) Influence of physical restraint on unplanned extubation of adult intensive care patients: a case-control study. Am J Crit Care 17: 408-415.

5. Bigwood S, Crowe M (2008) 'It's part of the job, but it spoils the job': a phenomenological study of physical restraint. Int J Ment Health Nurs 17: 215-222.

6. Chiba Y, Yamamoto-Mitani N, Kawasaki M (2012) A national survey of the use of physical restraint in long-term care hospitals in Japan. J ClinNurs 21: 1314-1326.

7. Benbenbishty J, Adam S, Endacott R (2010) Physical restraint use in intensive care units across Europe: the PRICE study. Intensive Crit Care Nurs 26: 241-245.

8. De Jonghe B, Constantin JM, Chanques G, Capdevila X, Lefrant JY, et al. (2013) Physical restraint in mechanically ventilated ICU patients: a survey of French practice. Intensive Care Med 39: 31-37.

9. Goethals S, Dierckx de Casterlé B, Gastmans C (2013) Nurses' ethical reasoning in cases of physical restraint in acute elderly care: a qualitative study. Med Health Care Philos 16: 983-991.

10. Goethals S, Dierckx de Casterlé B, Gastmans C (2012) Nurses' decisionmaking in cases of physical restraint: a synthesis of qualitative evidence. J AdvNurs 68: 1198-1210.

11. Bray K, Hill K, Robson W, Leaver G, Walker N, et al. (2004) British Association of Critical Care Nurses position statement on the use of restraint in adult critical care units. NursCrit Care 9: 199-212.

12. da Silva PS, Fonseca MC (2012) Unplanned endotracheal extubations in the intensive care unit: systematic review, critical appraisal, and evidencebased recommendations. AnesthAnalg 114: 1003-1014.

13. Jarachovic M, Mason M, Kerber K, McNett M (2011) The role of standardized protocols in unplanned extubations in a medical intensive care unit. Am J Crit Care 20: 304-311. 